\title{
A method to detect single-nucleotide polymorphisms accounting for a linkage signal using covariate-based affected relative pair linkage analysis
}

\author{
Yeunjoo E Song ${ }^{1 *}$, Junghyun Namkung ${ }^{1}$, Robert W Shields ${ }^{1,2}$, Daniel J Baechle ${ }^{1}$, Sunah Song ${ }^{1,2}$, Robert C Elston ${ }^{1}$
}

From Genetic Analysis Workshop 17

Boston, MA, USA. 13-16 October 2010

\begin{abstract}
We evaluate an approach to detect single-nucleotide polymorphisms (SNPs) that account for a linkage signal with covariate-based affected relative pair linkage analysis in a conditional-logistic model framework using all 200 replicates of the Genetic Analysis Workshop 17 family data set. We begin by combining the multiple known covariate values into a single variable, a propensity score. We also use each SNP as a covariate, using an additive coding based on the number of minor alleles. We evaluate the distribution of the difference between LOD scores with the propensity score covariate only and LOD scores with the propensity score covariate and a SNP covariate. The inclusion of causal SNPs in causal genes increases LOD scores more than the inclusion of noncausal SNPs either within causal genes or outside causal genes. We compare the results from this method to results from a family-based association analysis and conclude that it is possible to identify SNPs that account for the linkage signals from genes using a SNP-covariate-based affected relative pair linkage approach.
\end{abstract}

\section{Background}

Owing to the complexity of the genetic models underlying complex traits, model-free linkage methods, which do not require the specification of a disease model, are a popular choice. With these methods, inclusion of covariates increases the power to detect linkage [1], provided that the covariates reflect underlying locus heterogeneity. The method allows the genetic relative risk to depend on the covariate so that, in effect, the allele sharing at the marker locus differs for different values of the covariate. A general conditional-logistic model developed by Olson [2] provides a unified framework to incorporate covariates, and this model is implemented in LODPAL (SAGE, version 6.1.0) [3]. A modified oneparameter model has been proposed [4], so that only one additional parameter per covariate is required.

\footnotetext{
* Correspondence: yeunjoo.song@case.edu

'Department of Epidemiology and Biostatistics, Case Western Reserve

University, 10900 Euclid Avenue, Cleveland, OH 44106, USA

Full list of author information is available at the end of the article
}

To identify single-nucleotide polymorphisms (SNPs) that may explain the observed linkage signals, several researchers have developed methods for an affected pair analysis [5-10] and for quantitative trait linkage analysis [11]. Among these studies, Houwing-Duistermaat et al. [8] proposed using Olson's conditional-logistic model with a genotype-based covariate to explain the linkage signals. They applied the method to three SNPs and five markers in the Genetic Analysis Workshop 14 data, and they confirmed a SNP that explained a linkage peak. However, the statistical properties of this method still need to be studied. The large numbers of SNPs from exome sequencing data, along with the identical-bydescent (IBD) allele sharing from fully informative markers in the Genetic Analysis Workshop 17 (GAW17) data set [12], provide a good opportunity to evaluate this approach; hence our purpose here is to evaluate this new method in depth.

(c) 2011 Song et al; licensee BioMed Central Ltd. This is an open access article distributed under the terms of the Creative Commons Attribution License (http://creativecommons.org/licenses/by/2.0), which permits unrestricted use, distribution, and reproduction in any medium, provided the original work is properly cited. 


\section{Methods}

\section{Phenotype data}

We analyzed all 200 replicates of the GAW17 family data set. The binary affected status was analyzed as the main trait of interest, and the affected relative pairs from all eight extended pedigrees were used.

Based on the knowledge of the underlying simulating model, we included Age, Sex, and Smoking status as covariates in all analyses. Using the method of Doan et al. [13], we combined these three covariates into a single variable, a propensity score (PS), as a means of allowing for multiple covariates with the addition of only 1 degree of freedom ( $\mathrm{df}$ ). In each replicate, the PS values were estimated by taking the predicted probability of being affected, given the set of covariates, after fitting a logistic regression of affection status on the given covariates with all 698 individuals using $R$, version $2.10 .1[14]$.

\section{Marker data}

We used the IBD sharing values for the 3,205 genes from 22 autosomal chromosomes. After removing the SNPs with no variability in the data set or with no LOD score result from LODPAL, the average number of SNPs remaining for the analysis in each replicate was 9,069 out of 24,487 total SNPs. Among these 9,069 SNPs, 8,912 were in noncausal genes, 126 were noncausal SNPs in causal genes, and 31 were causal SNPs in causal genes. Each gene contained 1 to 231 SNPs. Using an additive coding based on the number of minor alleles, we recoded each SNP as 0,1 , or 2 .

\section{Analysis}

In the general conditional-logistic model by Olson [2], the likelihood ratio (LR) for a relative pair $r$ is written:

$$
\mathrm{LR}=\frac{\sum_{i=0,1,2} \lambda_{i} \hat{f}_{r i}}{\sum_{i=0,1,2} \lambda_{i} f_{r i}}
$$

where $\lambda_{i}$ is the relative risk of disease for an individual who shares $i$ alleles IBD with an affected relative, $f_{r i}$ is the prior probability that a pair will share $i$ alleles IBD, and $\hat{f}_{r i}$ is the estimated probability that a pair will share $i$ alleles IBD conditional on available marker data. The model is parameterized in terms of the logarithms of relative risk, so:

$$
\lambda_{i}=\exp \left(\beta_{i}+\sum_{j=1}^{K} \delta_{i j} x_{j}\right)
$$

where the $\delta_{i j}$ are the parameters associated with the covariate $x_{j}$, with $\beta_{0}$ and $\delta_{0}=0$. We use a one-parameter model so that only one additional parameter is estimated for each included covariate. The asymptotic distribution of the LR statistics (i.e., $4.605 \times$ LOD score) for the one-parameter model is a 50:50 mixture of a chi-square distribution with $K \mathrm{df}$ and a chi-square distribution with $K+1 \mathrm{df}$ when there are $K$ covariates in the model and the relative pairs are independent.

The first model in our analysis includes one covariate PS, so:

$$
\lambda_{i}=\exp \left(\beta_{i}+\delta_{i} \mathrm{PS}\right)
$$

The second model included two covariates, PS and a SNP, incorporating the SNPs in a gene one by one as an additional covariate, so:

$$
\lambda_{i}=\exp \left(\beta_{i}+\delta_{1 i} \mathrm{PS}+\delta_{2 i} \mathrm{SNP}\right)
$$

We evaluate the LOD score increases from the first model to the second model (LodDiff) to detect SNPs that differentially account for the linkage signals.

In each replicate, the LodDiff values are calculated for all available SNPs. Then, the mean LodDiff values are calculated for three different groups of SNPs: SNPs in noncausal genes, noncausal SNPs in causal genes, and causal SNPs in causal genes. The distributions of these mean LodDiff values over 200 replicates are compared. Again in each replicate, all SNPs are sorted and divided into 10 equal partitions (deciles) according to their LodDiff values, and the proportion of true causal SNPs within each partition is checked. We report the mean proportion values over 200 replicates.

To conduct family-based association analysis using the residuals obtained from the logistic regression model with Age, Sex, and Smoking as covariates, we use the ASSOC program in SAGE (version 6.1.0). ASSOC performs a likelihood-based regression unconditional on parental genotype. The analysis model includes a SNP as a fixed effect and a polygenic component as a random effect. To account for the nonnormal distribution of the residuals, we apply the George-Elston transformation. The $-\log (p$-value $)$ is summarized in the same way as the LodDiff value was from 200 replicates.

\section{Results and discussion}

In Figure 1 we plot the mean LOD scores from 200 replicates for each SNP for both models. In the first model without a SNP covariate, several genes in chromosomes 4 and 6 were significant (LOD score >3.0), and the inclusion of an additional SNP covariate increased the LOD score substantially in the second model. In Figure 2 the density plots of observed LR statistics from these mean LOD scores using SNPs in noncausal genes are compared with the theoretical 


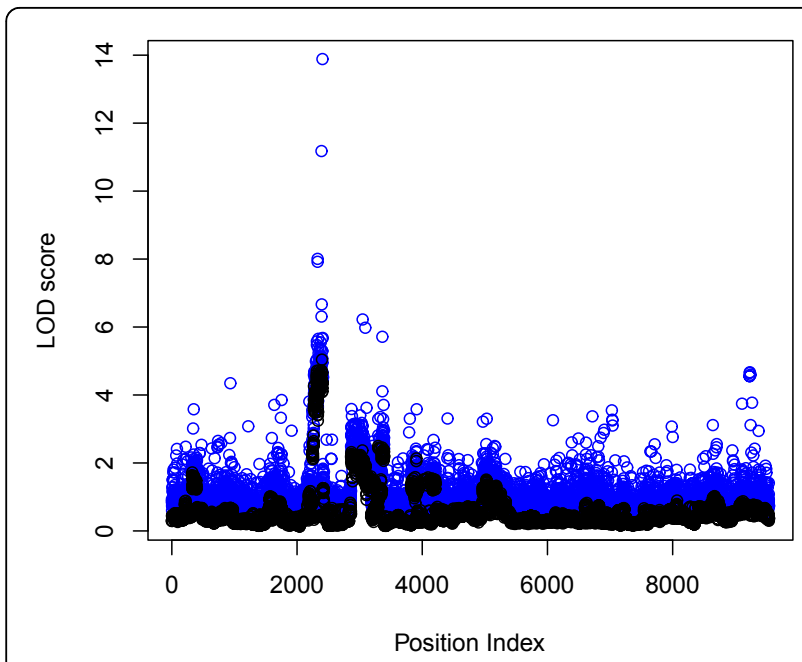

Figure 1 LOD scores from the model without SNP covariate and the model with SNP covariate. LOD score is plotted against SNP location. The first model without a SNP covariate is plotted in black, and the second model with a SNP covariate is plotted in blue.

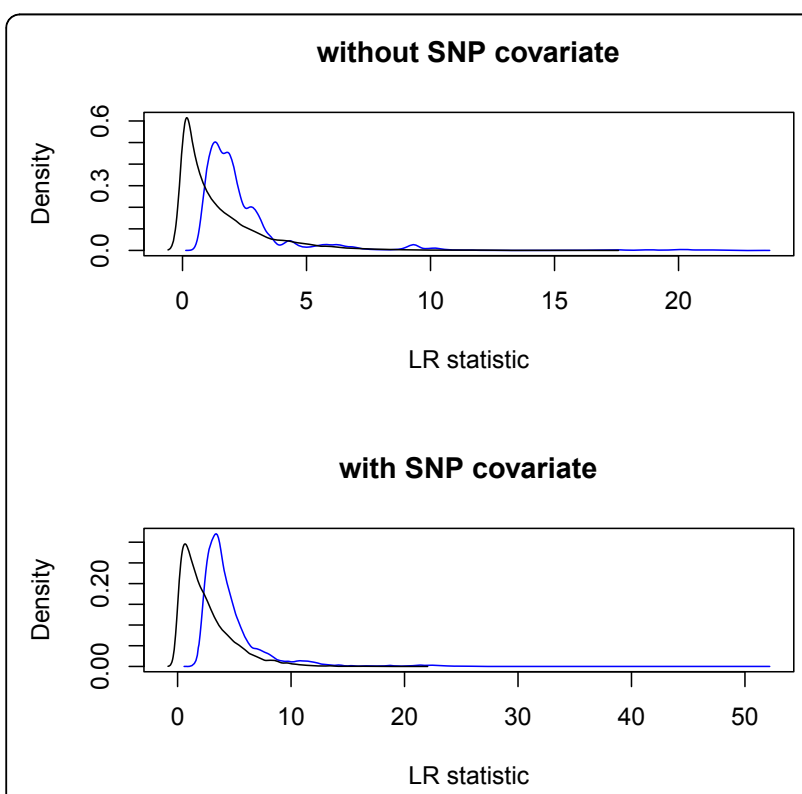

Figure 2 Observed LR statistics and theoretical mixture distribution. The density of observed LR statistics from LOD scores using SNPs in noncausal genes is plotted. For the first model without a SNP covariate, the theoretical distribution is a 50:50 mixture of a chi-square distribution with $1 \mathrm{df}$ and a chi-square distribution with $2 \mathrm{df}$ because we have one covariate. For the second model with a SNP covariate, the theoretical distribution is a 50:50 mixture of a chi-square distribution with $2 \mathrm{df}$ and a chi-square distribution with $3 \mathrm{df}$ because we have two covariates. The blue curve is for the observed LR statistics; the black curve is for the theoretical values.

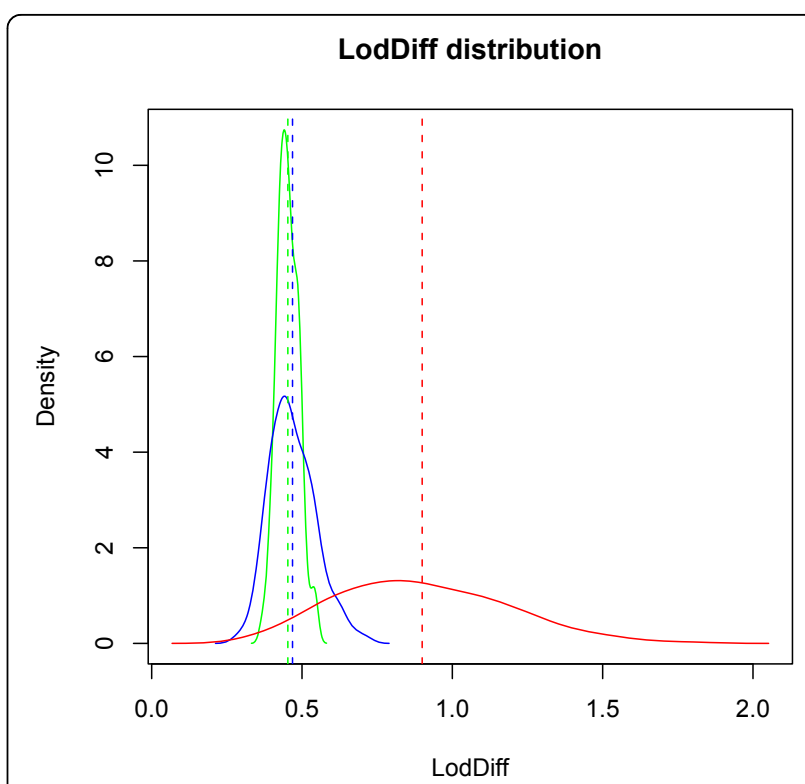

Figure 3 Density plots of LodDiff values. The distributions of LodDiff values for three groups of SNPs: noncausal SNPs in

noncausal genes (green), noncausal SNPs in causal genes (blue), and causal SNPs in causal genes (red). The dashed line in the same color as the curve indicates the mean location for each distribution.

50:50 mixture of a chi-square distribution with $1 \mathrm{df}$ and a chi-square distribution with $2 \mathrm{df}$ for the first model and with a 50:50 mixture of a chi-square distribution with $2 \mathrm{df}$ and a chi-square distribution with $3 \mathrm{df}$ for the second model.

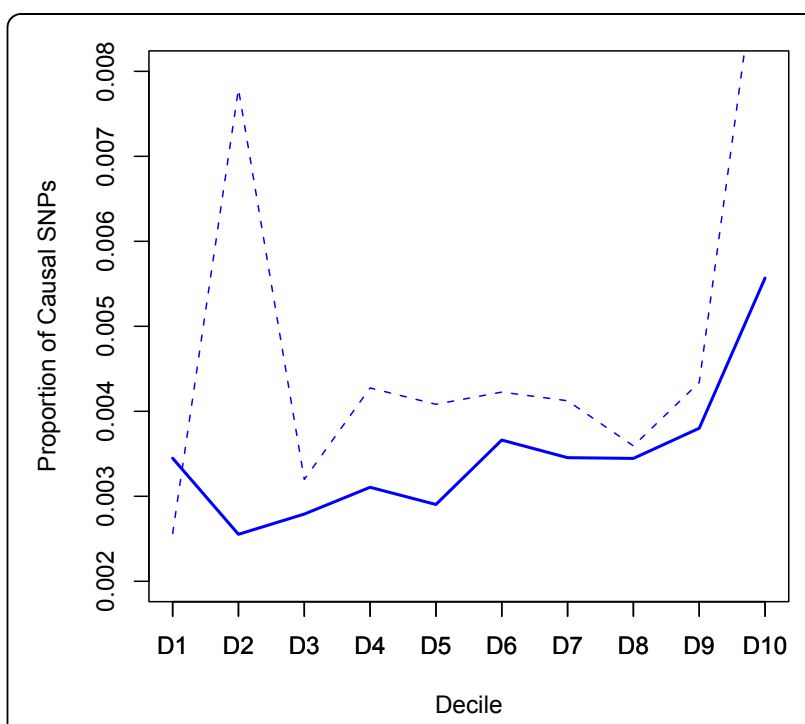

Figure 4 Proportions of causal SNPs in the LodDiff distribution. The plot shows the proportions of the causal SNPs in each decile of the sorted LodDiff values from the linkage analysis (solid line) and of the sorted $-\log (p$-value) from the family-based association analysis (dashed line). 
Table 1 Causal SNPs within the top 5\% of SNPs

\begin{tabular}{lcccccc}
\hline Chromosome & SNP & LodDiff & Gene & Minor allele frequency & Effect & $p$-value \\
\hline 4 & C4S4935 & 9.09 & VEGFC & 0.000717 & 1.35726 & 0.0000813 \\
6 & C6S2981 & 4.01 & VEGFA & 0.002152 & 1.20645 & 0.0000201 \\
10 & C10S3109 & 2.20 & SIRT1 & 0.000717 & 0.51421 & 0.0000112 \\
4 & C4S1878 & 1.45 & KDR & 0.164993 & 0.13573 & 0.0022138 \\
8 & C8S442 & 1.12 & LPL & 0.015782 & 0.49459 & NA \\
\hline
\end{tabular}

The information on five causal SNPs within the top 5\% of SNPs from the ordered LodDiff values are shown. The $p$-values in the last column are from the familybased association analysis.

The density plots of the mean LodDiff values for the three groups of SNPs are shown in Figure 3. From the distribution of LodDiff, we found that the inclusion of causal SNPs in causal genes increased the LOD scores more than the inclusion of noncausal SNPs did either within causal genes or outside causal genes. The overall mean LodDiff values were $0.45( \pm 0.04)$ for noncausal SNPs in noncausal genes, $0.47( \pm 0.07)$ for noncausal SNPs in causal genes, and $0.89( \pm 0.3)$ for causal SNPs in causal genes.

The plot of the proportions of the causal SNPs in each decile of the sorted mean LodDiff values, starting with the bottom decile, is shown in Figure 4. A clear tendency can be seen for this proportion to increase, implying that this approach may be able to detect causal SNPs. The proportions of causal SNPs in the ten deciles of $-\log (p$-values) from the family-based association analysis is presented with a dashed line for comparison.

From the ordered mean LodDiff values of SNPs, five causal SNPs (C4S4935, C6S2981, C10S3109, C4S1878, and C8S442) are included in the top 5\% (Table 1). Among these five causal SNPs, the top three SNPs (C4S4935, C6S2981, and C10S3109) are included in the top 1\%, and two of these SNPs are in the top $0.01 \%$. From the family-based association analysis, six causal SNPs (C10S3109, C6S2981, C4S4935, C4S1878, C9S444, and C13S523) are included in the top $5 \%$ of significant SNPs, four of which are among the SNPs identified by the covariate-based linkage analysis.

In addition, we checked the correlations between LodDiff and other properties of SNPs. The correlation between the LodDiff values and the number of additional SNPs in the gene being considered was 0.05 . The correlation with the minor allele frequency of the SNP included was 0.07 and 0.19 with the effect size. Our analysis did not consider the linkage disequilibrium structure. Linkage disequilibrium between SNPs within a gene and SNPs in different genes might affect the effectiveness of LodDiff. Further work is needed to investigate this matter.

\section{Conclusions}

We investigated the possibility of identifying SNPs that account for the linkage signals coming from genes using a covariate-based affected relative pair linkage approach. Further research is needed to study the statistical properties and the empirical null distribution to evaluate the significance of any result.

\section{Acknowledgments}

The Genetic Analysis Workshop is supported by National Institutes of Health grant R01 GM031575. Some of the results in this paper were obtained by using the program package SAGE, which is supported by a U.S. Public Health Service Resource Grant (RR03655) from the National Center for Research Resources.

This article has been published as part of BMC Proceedings Volume 5 Supplement 9, 2011: Genetic Analysis Workshop 17. The full contents of the supplement are available online at http://www.biomedcentral.com/1753$6561 / 5$ ? issue $=$ S9.

\section{Author details}

${ }^{1}$ Department of Epidemiology and Biostatistics, Case Western Reserve University, 10900 Euclid Avenue, Cleveland, OH 44106, USA. ${ }^{2}$ Department of Electrical Engineering and Computer Science, Case Western Reserve University, 10900 Euclid Avenue, 321 Glennan Building, Cleveland, OH 44106, USA.

\section{Authors' contributions}

YES conceived and designed the study, performed the analyses, and drafted the manuscript. JN carried out association analyses and helped draft and revise the manuscript. RWS helped with data preparation and edited the manuscript. DJB edited the manuscript and helped revise the manuscript. SS helped with visualization of the results. RCE participated in the design of the study and revised the manuscript. All authors read and approved the final manuscript.

\section{Competing interests}

The authors declare that there are no competing interests.

\section{Published: 29 November 2011}

\section{References}

1. Greenwood CMT, Bull SB: Analysis of affected sib pairs, with covariateswith and without constraints. Am J Hum Genet 1999, 64:871-885.

2. Olson JM: A general conditional-logistic model for affected-relative-pair linkage studies. Am J Hum Genet 1999, 65:1760-1769.

3. SAGE Project: SAGE: Statistical Analysis for Genetic Epidemiology. [http:// darwin.cwru.edu/sage].

4. Goddard KAB, Witte JS, Suarez BK, Catalona WJ, Olson JM: Model-free linkage analysis with covariates confirms linkage of prostate cancer to chromosomes 1 and 4. Am J Hum Genet 2001, 68:1197-1206.

5. Horikawa Y, Oda N, Cox NJ, Li X, Orho-Melander M, Hara M, Hinokio Y, Lindner TH, Mashima $H$, Schwarz PE, et al: Genetic variation in the gene encoding calpain-10 is associated with type 2 diabetes mellitus. Nat Genet 2000, 26:163-175.

6. Sun L, Cox NJ, McPeek MS: A statistical method for identification of polymorphisms that explain a linkage result. Am J Hum Genet 2002, 70:399-411. 
7. Li C, Scott LJ, Boehnke M: Assessing whether an allele can account in part for a linkage signal: the Genotype-IBD Sharing Test (GIST). Am J Hum Genet 2004, 74:418-431.

8. Houwing-Duistermaat J, Uh H, Lebrec J, Putter H, Hsu L: Modeling the effect of an associated single-nucleotide polymorphism in linkage studies. BMC Genet 2005, 6(suppl 1):S46.

9. Biernacka JM, Cornell HJ: Exploring causality via identification of SNPs or haplotypes responsible for a linkage signal. Genet Epidemiol 2007, 31:727-740.

10. Chen MH, Eerdewegh PV, Vincent QB, Alcais A, Abel L, Dupuis J: Evaluation of approaches to identify associated SNPs that explain the linkage evidence in nuclear families with affected siblings. Hum Hered 2010, 69:104-119.

11. Almasy L, Blangero J: Exploring positional candidate genes: linkage conditional on measured genotype. Behav Genet 2004, 34:173-177.

12. Almasy LA, Dyer TD, Peralta JM, Kent JW Jr, Charlesworth JC, Curran JE, Blangero J: Genetic Analysis Workshop 17 mini-exome simulation. BMC Proc 2011, 5:suppl 9-S2.

13. Doan BQ, Sorrant AJM, Frangakis CE, Bailey-Wilson JE, Shugart YY: Covariate-based linkage analysis: application of a propensity score as the single covariate consistently improves power to detect linkage. Eur J Hum Genet 2006, 14:1018-1026.

14. R Development Core Team: R: A Language and Environment for Statistical Computing. Vienna, R Foundation for Statistical Computing; 2008 [http://www.R-project.org].

doi:10.1186/1753-6561-5-S9-S84

Cite this article as: Song et al: A method to detect single-nucleotide polymorphisms accounting for a linkage signal using covariate-based affected relative pair linkage analysis. BMC Proceedings 2011 5(Suppl 9): 584.

\section{Submit your next manuscript to BioMed Central and take full advantage of:}

- Convenient online submission

- Thorough peer review

- No space constraints or color figure charges

- Immediate publication on acceptance

- Inclusion in PubMed, CAS, Scopus and Google Scholar

- Research which is freely available for redistribution

Submit your manuscript at www.biomedcentral.com/submit 